\title{
PERFIL DEMOGRÁFICO DE PACIENTES COM INFARTO AGUDO DO MIOCÁRDIO NO BRASIL: REVISÃO INTEGRATIVA
}

DEMOGRAPHIC PROFILE OF PATIENTS WITH ACUTE MYOCARDIAL INFARCTION IN BRAZIL: INTEGRATIVE REVIEW

PERFIL DEMOGRÁFICO DE PACIENTES CON INFARTO AGUDO DE MIOCARDIO EN BRASIL: REVISIÓN INTEGRADORA

Francisco Ariel Santos da Costa

Fabiene Lima Parente ${ }^{2}$

Maria Sinara Farias 3

Fabiara Lima Parente 4

Paloma Custódio Francelino 5

Layanny Teles Linhares Bezerra ${ }^{6}$

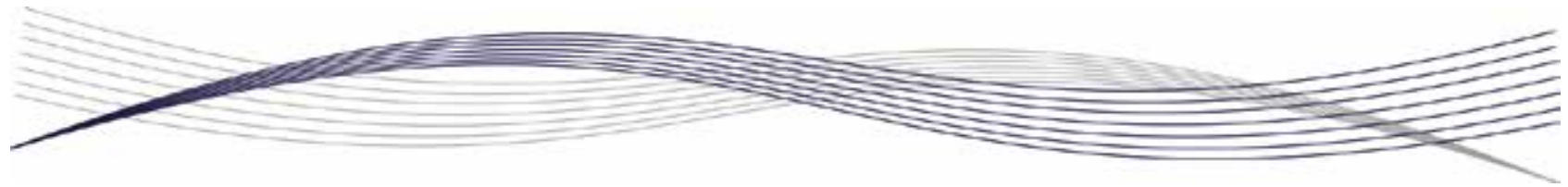

Palavras-chave: Infarto Agudo do Miocárdio; Doenças Cardiovasculares; Perfil de Saúde; Demografia.

Keywords:

Acute Myocardial Infarction; Cardiovascular Diseases; Health Profile; Demography.

Palabras clave: Infarto Agudo de Miocardio; Enfermedades Cardiovasculares; Perfil de Salud; Demografía.

Submetido: $18 / 09 / 2018$

Aprovado: 22/11/2018

Autor(a) para Correspondência: Francisco Ariel Santos da Costa Av. Geraldo Rangel, 715 Sobral (CE) CEP: 62042-240 E-mail: ariel.costa@stacasa.com.br

\section{RESUMO}

0 infarto agudo do miocárdio (IAM) é um evento cardiovascular grave que afeta as artérias coronárias e pode levar a morte se não diagnosticado $e$ tratado em tempo hábil. No Brasil, prevê-se que o IAM se torne a principal causa isolada de morte em 2020. Este artigo traça o perfil demográfico de pacientes com IAM por meio de pesquisa bibliográfica e descritiva à luz da literatura brasileira. Constatou-se que as regiões geográficas do Brasil mantêm a tendência internacional em relação ao perfil clínico dos pacientes com IAM, com predominância do sexo masculino e idade entre 56 e 58 anos. No entanto, os desfechos clínicos e as taxas de mortalidade inter-regionais se mostram heterogêneas, com maior divergência entre o Sudeste e o Nordeste - diminuição e aumento, respectivamente, das taxas de insucesso no manejo do paciente com IAM ao longo dos últimos anos, reforçando a necessidade de políticas públicas em saúde que favoreçam $e$ contribuam para o acesso e tratamento de qualidade no Nordeste, primando pelo princípio da equidade no Sistema Único de Saúde (SUS).

\footnotetext{
1. Enfermeiro no Serviço de Hemodinâmica do Hospital do Coração de Sobral. Sobral (CE), Brasil.

2. Enfermeira. Coordenadora de Enfermagem e Qualidade Hospitalar do Hospital do Coração de Sobral. Sobral (CE), Brasil.

3. Enfermeira na Unidade de Terapia Intensiva (UTI) do Hospital do Coração de Messejana. Fortaleza (CE), Brasil.

4. Enfermeira. Gerente de Risco do Hospital do Coração de Sobral. Sobral (CE), Brasil.

5. Enfermeira. Coordenadora do Serviço de Hemodinâmica do Hospital do Coração de Sobral. Sobral (CE), Brasil.

6. Enfermeira. Membro da Organização de Procura de Órgãos da Santa Casa de Misericórdia de Sobral. Sobral (CE), Brasil
} 


\section{ABSTRACT}

Acute myocardial infarction (AMI) is a serious cardiovascular event that affects the coronary arteries and it can lead to death if not diagnosed and treated in a timely manner. In Brazil, AMI is expected to become the leading isolated cause of death in 2020. This article traces the demographic profile of AMI patients through bibliographic research and descriptive in the light of Brazilian literature. It was found that the Brazilian geographic regions maintain the international trend in relation to the clinical profile of AMI patients, with predominance of men and age between 56 and 58 years. However, clinical outcomes and interregional mortality rates are heterogeneous, with greater divergence between the Southeast and Northeast - decrease and increase, respectively, in the failure rates in managing AMI patients over the last years, reinforcing the need for public health policies that favor and contribute to provide access and good-quality treatment in the Northeast, complying with the principle of equity in the Brazilian National Health System (SUS).

\section{RESUMEN}

El infarto agudo de miocardio (IAM) es un evento cardiovascular grave que afecta a las arterias coronarias y puede provocar la muerte si no se diagnostica y trata de manera oportuna. En Brasil, se espera que el AMI se convierta en la principal causa aislada de muerte en 2020. Este artículo rastrea el perfil demográfico de los pacientes con IAM a través de investigación bibliográfica y descriptivo a la luz de la literatura brasileña. Se constató que las regiones geográficas brasileñas mantienen la tendencia internacional con relación al perfil clínico de pacientes con IAM, con predominio del sexo masculino y edad entre 56 y 58 años. Sin embargo, los resultados clínicos y las tasas de mortalidad interregional son heterogéneas, con una mayor divergencia entre el Sudeste y el Nordeste - disminución y aumento, respectivamente, de las tasas de fracaso en el manejo de pacientes con IAM en los últimos años, lo que refuerza la necesidad de políticas de salud pública que favorezcan y contribuyan al acceso y tratamiento de calidad en el Nordeste, primando por el principio de la equidad en el Sistema Único de Salud Brasileño (SUS).

\section{INTRODUÇÃO}

As doenças cardiovasculares são alterações que afetam as funções responsáveis por carrear oxigênio e nutrir os tecidos celulares para que estes possam desempenhar de modo eficaz suas funções. Tais doenças são consideradas um grande problema de saúde pública em nível global. Pode-se destacar como mais relevantes: síndrome coronariana aguda (SCA); insuficiência cardíaca (IC); infarto agudo do miocárdio (IAM); doenças valvulares; arritmias; e hipertensão arterial sistêmica (HAS) ${ }^{1}$.

0 IAM pode ser definido como uma afecção isquêmica abrupta que reflete a morte dos miócitos cardíacos, causada por um desequilíbrio entre oferta e demanda de nutrientes ao tecido, consequente à obstrução do fluxo coronariano, podendo ser transitória ou permanente. Além da morte, pode deixar sequelas no indivíduo, gerando repercussões físicas, psicológicas e sociais. 0 paciente com IAM necessita de intervenção imediata e assistência à saúde após a alta hospitalar, para diminuir as chances de complicações e agravamento da doença a curto e médio prazo². Quando não diagnosticadas e tratadas de modo eficaz, as coronariopatias podem contribuir para o surgimento de outros agravos à saúde, como IC, pela diminuição prolongada na perfusão do músculo cardíaco ${ }^{3}$.

Essa interrupção ou diminuição súbita do fluxo sanguíneo por um tempo curto ou prolongado serão suficientes para causar a morte das células cardíacas. A morte tecidual cardíaca pode ser diagnosticada por meio do eletrocardiograma (ECG) e quando níveis sanguíneos de marcadores biológicos sensiveis e específicos como a troponina cardíaca e a creatinoquinase fração MB (CK MB) se encontram elevados. São múltiplos os fatores desencadeantes do processo de aterosclerose que levam ao surgimento e à progressão do IAM, relacionados a estilo de vida, dieta, uso de substâncias, hábitos diários, biotipo e fatores crônicos, congênitos e até emocionais. Estes podem levar a um evento agudo e evitável, denominado trigger (gatilho) quando ocorre abruptamente ${ }^{4}$.

Dados do Departamento de Informática do Sistema Único de Saúde (DataSUS) de 2013 revelam que o IAM foi a principal causa de morte por doença cardíaca no Brasil, com aumento de $48 \%$ entre 1996 e 2011 . Se essa tendência persistir, prevê-se que o IAM passará a ser a principal causa isolada de morte em 2020, 
um fator preocupante e que evidencia a necessidade de estratégias de intervenção e melhorias para essa parcela populacional ${ }^{5}$.

Desse modo, traçar o perfil demográfico de pacientes com IAM no Brasil em relação à sua região geográfica se mostra uma iniciativa válida para aprofundar e embasar conhecimentos sobre contextos do adoecimento cardiovascular, tendo em vista o crescente número de casos e internações hospitalares, bem como a importância da continuidade e atualização de estudos específicos.

\section{METODOLOGIA}

Trata-se de revisão integrativa orientada pela seguinte questão norteadora:

- Qual é o perfil demográfico de pacientes com diagnóstico de IAM no Brasil?

A busca foi realizada no portal da Biblioteca Virtual em Saúde (BVS) e nas bases de dados Lilacs e BDEnf.

Adotou-se como termo de busca, entre aspas, "infarto agudo do miocárdio", como consta na plataforma Descritores em Ciências da Saúde (DeCS). Os critérios de inclusão de artigos foram: versar sobre a temática; ser publicados no período de 2013 a 2017, no Brasil e em português; e estar disponíveis em texto integral, com acesso livre e gratuito. E os critérios de exclusão foram: artigos pagos; estudos repetidos; e pesquisas que não contemplavam a temática em questão.

A revisão integrativa teve 6 fases $^{6}$ para a busca e a análise:

1. Identificação do tema;

2. Estabelecimento dos critérios de inclusão e exclusão;

3. Categorização dos estudos;

4. Avaliação dos estudos incluídos;

5. Interpretação dos resultados; e

6. Sintese do conhecimento.

Inicialmente foram identificados 154.872 artigos. Com a aplicação dos critérios de inclusão e exclusão, restaram 54 artigos. Após leitura prévia dos resumos, estes foram caracterizados como: a) analisado; b) repetido; e c) fora da temática. (hegou-se, então, a um total de 18 artigos. 0s demais foram excluídos por não contemplar a temática de modo integral (31) ou por ser repetidos (5).

0 s estudos foram avaliados em suas respectivas categorias temáticas, já subdivididas, intercalando ideias e pensamentos afins entre os autores e quanto à temática inicial, de modo que se mostrassem coerentes diante da questão norteadora. Durante o estudo, respeitou-se a autonomia e a colaboração científica de cada autor, bem como seu direito de autoria, incluindo as devidas citações no corpo do texto e listando cada obra entre as referências consultadas, com vistas a respeitar todos os aspectos éticos envolvidos em uma revisão integrativa.

\section{RESULTADOS E DISCUSSÃO}

Por meio do acesso às bases de dados indicadas, do uso do termo de busca (caracterizado como abrangente) e da aplicação dos critérios de inclusão e exclusão, a amostra totalizou 18 artigos, caracterizados na Tabela 1.

Tabela 1 - Distribuição dos artigos por estado de realização da pesquisa.

\begin{tabular}{lcc}
\hline Estado & N & $\%$ \\
\hline Paraná & 1 & $5,53 \%$ \\
Rio Grande do Sul & 2 & $11,12 \%$ \\
Ceará & 1 & $5,53 \%$ \\
Santa Catarina & 1 & $5,53 \%$ \\
Bahia & 3 & $16,72 \%$ \\
Rio de Janeiro & 2 & $11,12 \%$ \\
Minas Gerais & 2 & $11,12 \%$ \\
São Paulo & 6 & $33,3 \%$ \\
\hline TOTAL & 18 & $100 \%$ \\
\hline
\end{tabular}

Fonte: Elaborada pelos autores.

Observa-se na Tabela 1 que o maior percentual de estudos sobre IAM analisados se refere ao estado de São Paulo (33,3\%), incluindo-se nesse grupo pesquisas bibliográficas, estudos de coorte e estudos randomizados.

Em segundo lugar, na região Nordeste, a maior frequência ocorreu na Bahia $(16,72 \%)$. 0 Ceará ocupa a $4^{\text {a }}$ e última posição, com o mesmo percentual do Paraná (onde ocorreu a primeira angioplastia coronariana no Brasil, em 1979) e de Santa Catarina. Vale ressaltar que os únicos estados do Nordeste a figurar no ranking foram Bahia e Ceará. Todos os demais estados se encontram no Sul e Sudeste do Brasil.

A Tabela 2 caracteriza o período de publicação dos artigos. 
Tabela 2 - Distribuição dos artigos por ano de publicação.

\begin{tabular}{lcc}
\hline Ano de publicação & N & $\%$ \\
\hline 2013 & 7 & $38,9 \%$ \\
2014 & 2 & $11,1 \%$ \\
2015 & 2 & $11,1 \%$ \\
2016 & 5 & $27,8 \%$ \\
2017 & 2 & $11,1 \%$ \\
\hline TOTAL & 18 & $100 \%$ \\
\hline
\end{tabular}

Fonte: Elaborada pelos autores.

Observa-se na Tabela 2 que 2013 foi o ano com maior quantidade de publicações $(38,9 \%)$, seguido por $2016(27,8 \%)$. Ressalta-se a importância do incentivo por parte das entidades de pesquisa para obter um levantamento mais preciso da situação nacional de adoecimento cardiovascular. Mostrase necessário acompanhar os problemas de saúde pública com base na dinâmica do processo saúdedoença em diferentes cortes populacionais.

As discussões dos achados dos artigos se distribuem em três grandes categorias, considerando a principal temática e as evidências de pesquisa: a) Mortalidade e desfechos clínicos do IAM no Brasil (6 artigos); b) Perfis clínicos de pacientes com IAM nas regiões demográficas (5 artigos); e c) Problemas observados nas redes de saúde brasileiras (7 artigos).

\section{Mortalidade e desfechos clínicos por infarto agudo do miocárdio no Brasil}

A primeira categoria temática sobre a mortalidade e os demais desfechos clínicos do IAM no Brasil evidencia os índices de morbimortalidade pelo agravo entre pacientes atendidos em emergências cardiológicas e internados em hospitais de referência em todo o território brasileiro.

Um estudo ${ }^{7}$ que analisou o efeito idade-período e coorte (age-period and cohort - APC) nas regiões geográficas do Brasil entre 1980 e 2009 constatou redução do risco de mortalidade por IAM em todo o país entre indivíduos nascidos a partir da década de 1940, exceto no Nordeste - onde esse índice aumentou, ao contrário da tendência nacional.

Uma segunda pesquisa ${ }^{8}$ sobre o tema confirmou a redução da mortalidade por IAM na região Sul. No início do estudo, indicava-se uma estimativa de aumento do número de mortes em relação aos

\author{
No Brasil, ainda se \\ notam elevadas taxas \\ de morbimortalidade \\ por IAM nos contextos \\ intra e extra- \\ hospitalar...
}

últimos 12 anos, mas isso não se confirmou e houve redução de $38 \%$ da mortalidade por IAM ao comparar os dados de 2009 aos de 1998.

Tais estudos reforçam a redução da mortalidade, a melhoria da qualidade da assistência e o aumento da sobrevida dos pacientes com coronariopatia acometidos por IAM em um recorte de 20 a 30 anos (período de implementação do SUS), no entanto, isso não ocorre de modo homogêneo no país, notando-se disparidade entre as regiões Nordeste e Sul ${ }^{7-8}$.

Ressalta-se, também, que um terceiro estudo9, realizado em nível nacional, sobre as terapêuticas comprovadamente úteis disponibilizadas aos pacientes com isquemia aguda, tendo por base as diretrizes nacionais de recomendação e boas práticas, reforça os dados de estudos anteriores da mortalidade por IAM nas regiões brasileiras, que indicam diminuição no Sul e Sudeste, manutenção no Centro-0este e aumento no Nordeste.

No Brasil, ainda se notam elevadas taxas de morbimortalidade por IAM nos contextos intra e extra-hospitalar, seja pela dificuldade de acesso aos serviços especializados após os primeiros sinais de progressão do quadro ou pelo retardo na busca de tratamento ambulatorial em nível de atenção primária à saúde (APS), seja pela falta de orientação especifica ou mesmo ausência de atendimento eficaz no serviço de saúde público, o qual atende a maioria da população brasileira em todos os níveis de atenção. Contudo, essas situações não são observadas de modo homogêneo em todo o território nacional, havendo disparidades entre as diversas regiões e seus contextos sociais ${ }^{7-9}$.

\section{Perfis clínicos de pacientes com infarto agudo do miocárdio nas regiões geográficas}

A segunda categoria temática aborda o perfil clínico de pacientes com IAM em relação às regiões demográficas do país, correlacionando cada um 
deles às realidades e especificidades regionais no que diz respeito a acesso aos serviços de saúde, condições sanitárias e demais fatores que interferem diretamente no processo saúde-doença.

Uma pesquisa ${ }^{10}$ realizada em hospital cardiológico da Bahia, cujo propósito era levantar a avaliação de enfermeiros acerca do risco coronariano dos pacientes internados, caracterizou o perfil clínico da população estudada assim: $59,5 \%$ de indivíduos do sexo masculino, $64,3 \%$ se consideram brancos, e 50,8 anos foi a média de idade. Quanto à avaliação de exames laboratoriais, sinais vitais e antecedentes, observou-se que: $40,5 \%$ apresentaram doses elevadas de colesterol, 52,4\% apresentaram níveis pressóricos elevados e $35,7 \%$ eram tabagistas.

Um estudo clínico ${ }^{11}$ realizado em instituto cardiológico do Rio Grande do Sul, com vistas a traçar a etiologia e as demais características de pacientes com diagnóstico de IAM (tipo 2), evidenciou média de idade de 50,6 anos, 58,1\% de indivíduos do sexo masculino, $80,6 \%$ se considerando brancos, $58,1 \%$ portadores de HAS, $29 \%$ dislipidêmicos e $32,3 \%$ tabagistas.

Entretanto, nota-se em estudos mais recentes (de 2016 e 2015), em regiões geograficamente opostas, Nordeste (Bahia) e Sul (Rio Grande do Sul), que os perfis dos pacientes se mantêm muito próximos, tendo por base sexo, idade, tabagismo e HAS, porém, diferem quanto a cor da pele, com maior percentual de indivíduos brancos no Sul do país, o que se relaciona aos fatores históricos (migratórios e de colonização) e aos aspectos dislipidêmicos, para os quais o Nordeste apresenta percentual $11,5 \%$ superior aos do Sul10-11.

Outro estudo ${ }^{9}$, que compara as regiões demográficas em nível nacional, enfoca os parâmetros supracitados e evidencia que, na região Sudeste, a média de idade entre pacientes hospitalizados com IAM foi de 63,1 anos; $66,4 \%$ dos indivíduos era do sexo masculino; $74,7 \%$ se consideravam brancos; $70,9 \%$ apresentavam quadro de HAS e $34,6 \%$ de dislipidemia (DLP); e havia tabagistas atuais ou prévios em $55,4 \%$ dos casos. Já na região Nordeste, a média de idade foi de 65,6 anos; $52,6 \%$ dos indivíduos eram do sexo masculino; $57,2 \%$ se consideraram brancos; $69,3 \%$ apresentavam quadro de HAS e $39,3 \%$ de DLP; e $45,1 \%$ dos casos envolviam tabagistas.

Observou-se a partir desses dados que distintas regiões geográficas também apresentaram alterações quanto ao perfil dos pacientes analisados, o que pode estar diretamente relacionado a fatores não

\author{
..os perfis dos \\ pacientes se mantêm \\ muito próximos, tendo \\ por base sexo, idade, \\ tabagismo e HAS...
}

só genéticos, mas culturais. No entanto, o perfil clássico em que se observa a predominância do sexo masculino se manteve equiparado nas duas regiões, com aumento de idade dos pacientes do Nordeste ${ }^{9-10}$.

Sobre os fatores culturais supracitados, um estudo realizado em hospital especializado do interior do Ceará com mulheres com diagnóstico de IAM, após procedimento de angioplastia, evidenciou estranhamento das pacientes em relação ao ambiente físico e às rotinas inerentes a o setor: banho no leito e manutenção de decúbito dorsal com cabeceira a zero grau (em casos de abordagem por via femoral durante a permanência de introdutor arterial), luminosidade do setor, ausência de referência temporal/espacial e higienização oral com uso de antissépticos líquidos (enxaguantes bucais) ${ }^{12}$.

Vale a reflexão tanto acerca das especificidades inerentes às regiões como das rotinas e normas, baseadas em evidências para a segurança do paciente, que também podem ser distintas em diferentes áreas geográficas e hospitais brasileiros. Atribui-se, ainda, a necessidade das informações disponibilizadas aos pacientes durante a internação levarem em consideração seu nível de letramento funcional em saúde, o que pode influenciar tanto o tratamento hospitalar e pós-hospitalar como o seguimento ambulatorial.

Após avaliação realizada em hospital de referência cardiológica na região Sul do país, alguns autores $^{13}$ destacam a taxa de $20 \%$ de mortalidade intra-hospitalar em pacientes com IAM. Os autores associam o pior prognóstico dos pacientes com IAM sem supradesnivel do segmento ST ao acometimento da parede anterior e à evolução de IC a médio prazo nessa população.

Em um comparativo realizado com 1.150 pacientes com SCA em hospitais brasileiros, em todas as 5 regiões geográficas, evidenciou-se que $63,7 \%$ eram do sexo masculino, com idade média de 63,1 anos e $69,3 \%$ se consideram brancos. HAS e DLP foram observadas em $2 / 3$ dos pacientes na admissão 
hospitalar e mais da metade dos indivíduos referiu tabagismo atual ou prévio ${ }^{14}$.

Outro autor ${ }^{15}$ reforça a predominância do sexo masculino em estudo realizado durante 3 anos em Belo Horizonte sobre a linha de cuidado dos pacientes com IAM, chegando aos seguintes resultados: $63,3 \%$ (2009); 63,9\% (2010) e 62,0\% (2011). A média de idade foi de 60,7 anos.

Nota-se manutenção das taxas nacionais entre os estudos referentes à população com doenças cardiovasculares, no entanto, as nuances inter-regionais podem envolver a indicação de particularidades e divergências nos atendimentos e perfis clínicos dos pacientes com IAM no que diz respeito às regiões demográficas. Contudo, são alterações relativamente baixas em relação às características da população analisada, o que não confere ligação direta a esses dados nem explica por que as diferenças entre os desfechos clínicos são mais elevadas ao comparar regiões distintas, o que leva a crer que tais divergências estão ligadas mais fortemente aos processos de acesso e qualidade dos recursos do que às características isoladas do perfil da população $0^{13-15}$.

\section{Problemas observados nas redes de saúde brasileiras}

A terceira e última categoria temática versa sobre os processos logísticos e os problemas observados nas redes de saúde pública e privada, pois a garantia de qualidade da assistência pode estar diretamente ligada às condições monetárias e estruturais inerentes à gestão e à realidade prática desses serviços.

As redes de saúde brasileiras dividem-se basicamente em 2 grupos: a) SUS, que cobre toda a população do país com base em seus preceitos básicos, como a gratuidade e integralidade da assistência, formulado e regulamentado a partir da Constituição Federal de 1988; e b) Sistema de Saúde Suplementar (SSS), que engloba as iniciativas de empresas privadas de prestação de serviços, após a contratação de seus planos de saúde, nos diferentes níveis de atenção à saúde ${ }^{16}$.

Segundo os mesmos autores ${ }^{16}$, apenas os processos de adoecimento cardiovascular totalizam 1 milhão de internações por ano no SUS, resultando em gastos da ordem de $R \$ 1,9$ bilhão ( $5 \%$ da receita em saúde), considerando os dados anuais desde o início da década de 2000 .

\section{...os processos \\ de adoecimento cardiovascular \\ totalizam 1 milhão de internações por ano no SUS...}

Outros pesquisadores ${ }^{17}$ evidenciaram, em estudo desenvolvido em hospital público da Bahia, que $96,9 \%$ pacientes com quadro de angina pectoris, posteriormente diagnosticados ou não com IAM, realizaram o translado domicílio-hospital com meio de transporte próprio ou de familiares/amigos e $68 \%$ deles procuraram o hospital como primeira escolha após o surgimento dos sintomas. No entanto, apenas $33 \%$ foram internados com quadro confirmado de IAM ou SCA.

Em 2002, a mortalidade da população com IAM no Estado do Rio de Janeiro foi de 28,6\%, pois $1.241,8$ dos 4.435 dos pacientes atendidos com esse diagnóstico na rede pública evoluíram a óbito na mesma internação. 0 gasto diário com a internação hospitalar foi superior a $R \$ 905,00$ por paciente, com uso de tecnologias de moderada a alta complexidade, bem como de UTI, concluindo-se que os aspectos isolados de maior gravidade (óbito), maior custo e maior utilização de UTI por pacientes com diagnóstico de IAM não vêm sendo empregados de modo efetivo em todos os $\operatorname{casos}^{16-17}$.

Outros autores indicam ${ }^{4}$ indica um evento esportivo, especificamente a Copa do Mundo de Futebol, no Brasil, como desencadeador comprovado de eventos cardiovasculares, ressaltando a má estruturação dos estádios de futebol nacionais para o manejo correto desses possíveis eventos, aumentando o risco de complicações inerentes aos agravos.

Uma pesquisa ${ }^{18}$ realizada em hospital universitário público na cidade de São Paulo compara as taxas de mortalidade intra-hospitalar de pacientes com diagnóstico inicial de IAM em 2009 e 2010, consecutivamente pré e pós-implementação da chamada "rede de infarto", que capacitou unidades de apoio e instaurou estratégias para a melhoria do diagnóstico precoce e do transporte, além do aumento da agilidade das técnicas de reperfusão miocárdica. Observou-se considerável diminuição do percentual de óbitos nessa população, passando de 
$26,14 \%$ em 2009 para 7,31\% em 2010. CONCLUSÃO

Após a análise e quantificação das informações, a soma dos resultados afins e sua divisão pelo número de pesquisas possibilitou a obtenção das médias aritméticas, com vistas a traçar o perfil demográfico de pacientes com IAM proposto. Vale ressaltar que só foi possivel mensurar esses dados com base nas regiões Sul, Sudeste e Nordeste, pois as demais não foram contempladas pelos artigos selecionados.

$\mathrm{Na}$ região Sudeste, observou-se que a média de idade dos pacientes com IAM foi de 56,8 anos, ou seja, 1,4 ano a menos do que na região Nordeste (58,2 anos); no entanto, vale afirmar que tais dados se relacionam diretamente a procura e acesso aos serviços de saúde especializados disponíveis na região, tendo em vista que se trata de informações coletadas em contexto intra-hospitalar de referência. Contudo, há discreta variação de resultados entre o Sudeste e o Nordeste, regiões geograficamente opostas.

Constatou-se predominância do sexo masculino dentre os pacientes com IAM em ambas as regiões. 0 Sudeste tem índice maior $(62,2 \%)$ em comparação ao Nordeste $(59,5 \%)$. Também se nota discreta diferença entre as regiões e a manutenção das tendências nacional e internacional de predominância do sexo masculino nessa população.

Quanto aos desfechos clínicos, os estudos mostraram divergências no sucesso terapêutico e menores taxas de óbito nas regiões Sul e Sudeste em comparação ao Nordeste, onde se observa aumento dos indicadores de morbimortalidade.

Tendo em vista que o perfil clínico dos pacientes se mantém equiparado nas regiões com divergência significativa entre sucesso do tratamento e melhores desfechos clínicos, pode-se apontar que os problemas envolvidos não decorrem de fatores genéticos, mas daqueles relacionados a entraves de direcionamento e fluxo das políticas públicas e dos demais fatores de acesso inadequado aos serviços de saúde de referência e especializados.

As informações levantadas evidenciam a necessidade de contínua melhoria e aperfeiçoamento do acesso a serviços cardiovasculares, com vistas a adequar a realidade prática à demanda em nível nacional. Deve-se ressaltar, ainda, a importância da prevenção de doenças e da promoção da saúde na APS, para evitar o "estrangulamento" das redes de

\section{saúde brasileiras a longo prazo.}

\section{CONTRIBUIÇÃO DOS AUTORES}

Francisco Ariel Santos da Costa contribuiu com a realização da pesquisa, o delineamento do estudo e a redação do manuscrito. Fabiene Lima Parente, Maria Sinara Farias, Paloma Custódio Francelino e Layanny Teles Linhares Bezerra contribuíram com a redação e revisão crítica do manuscrito.

\section{REFERÊNCIAS}

1. Aehlert B. ACLS, suporte avançado de vida em cardiologia: emergência em cardiologia. Rio de Janeiro: Elsevier; 2013.

2. Silveira DS, Jaeger CP, Hatschbach L, Manenti ERF. Validação do escore TIMI de risco para infarto agudo com supradesnivelamento do Segmento ST. Int J Cardiovasc Sci [serial on the internet]. 2016 [cited 2018 Nov 28];29(3):189-97. Available from: http:// www.onlineijcs.org/sumario/29/pdf/v29n3a06.pdf

3. Viana PAS, Carneiro Neto JD, Novais CT, Guimarães IF, Lopes YS, Reis BC. Perfil de pacientes internados para tratamento de insuficiência cardíaca descompensada. Sanare (Sobral, Online) [serial on the internet]. 2018 [serial on the internet];17(1):15-23. Available from: https://sanare.emnuvens.com.br/ sanare/article/view/1218/649

4. Borges DGS, Monteiro RA, Schmidt A, Pazin-Filho A. Copa do Mundo de Futebol como desencadeador de eventos cardiovasculares. Arq Bras Cardiol [serial on the internet]. 2013 [cited 2018 Nov 28];100(6):54652. Available from: http://www.scielo.br/pdf/abc/ v100n6/aop 5334.pdf

5. Feres F, Costa RA, Siqueira D, Costa JR Chamié D, Staico R. Diretriz da Sociedade Brasileira de Cardiologia e da Sociedade Brasileira de Hemodinâmica e Cardiologia Intervencionista sobre intervenção coronária percutânea. Arq Bras Cardiol [serial on the internet]. 2017 [cited 2018 Nov 28];109(Suppl 1):1-81. Available from: http://www. scielo.br/pdf/abc/v109n1s1/0066-782X-abc-10901-s1-0001.pdf

6. Mendes KDS, Silveira RCCP, Galvão CM. Revisão integrativa: método de pesquisa para a incorporação de evidências na saúde e na enfermagem. Texto \& Contexto Enferm [serial on the internet]. 2008 [cited 2018 Nov 28];7(4):758-64. Available from: http://www.scielo.br/pdf/tce/v17n4/18.pdf

7. Santos J, Meira KC, Camacho AR, Salvador PTCO, Guimarães RM, Pierin AMG, et al. Mortalidade por infarto agudo do miocárdio no Brasil e suas regiões geográficas: análise do efeito da idade-períodocoorte. Ciênc Saúde Colet [serial on the internet]. 2018 [cited 2018 Nov 28];23(5):1621-34. Available from: http://www.scielo.br/pdf/csc/v23n5/14138123-csc-23-05-1621.pdf 
8. Baena CP, Olandoski M, Luhm KR, Constantini C0, Souza LCG, Neto JRF. Tendência de mortalidade por infarto agudo do miocárdio em Curitiba (PR) no período de 1998 a 2009 . Arq Bras Cardiol [serial on the internet]. 2014 [cited 2018 Nov 28];98 (3):2117. Available from: http://www.scielo.br/pdf/abc/ v98n3/v98n3a03.pdf

9. Nicolau JC, Franken M, Lotufo PA, Carvalho AC, Marin Neto JA, Lima, FG, et al. Utilização de terapêuticas comprovadamente úteis no tratamento da coronariopatia aguda: comparação entre diferentes regiões brasileiras. Análise do Registro Brasileiro de Síndromes Coronarianas Agudas (BRACE - Brazilian Registry on Acute Coronary Syndromes). Arq Bras Cardiol [serial on the internet]. 2014 [cited 2018 Nov 28];98(4):282-9. Available from: http:// www.scielo.br/pdf/abc/v98n4/v98n4a01.pdf

10. Rosa RS, Macêdo DA, Oliveira BG, Bomfim ES, Casotti CA, Prado IF. Evidências para o cuidado de enfermagem na avaliação do risco coronariano em pacientes hospitalizados. Rev Pesqui Cuid Fundam (Online) [serial on the internet]. 2016 [cited 2018 Nov 28];8(2):4460-71. Available from: http://www. seer.unirio.br/index.php/cuidadofundamental/ article/view/4647/pdf 1900

11. Schmidt MM, Quadros AS, Martinelli ES, Gottschall CAM. Prevalência, etiologia e características dos pacientes com infarto agudo do miocárdio tipo 2. Rev Bras Cardiol Invasiva [serial on the internet]. 2015 [cited 2018 Nov 28];23(2):119-23. Available from: https://www.sciencedirect.com/science/article/pii/ $\underline{\text { S0104184315000429 }}$

12. Ponte KMA, Silva LF. Cuidados de enfermagem a mulheres com infarto do miocárdio: promoção do conforto sociocultural pela pesquisa-cuidado. Rev Enferm UERJ [serial on the internet]. 2014 [cited 2018 Nov 28];22(6):808-14. Available from: http:// www.facenf.uerj.br/v22n6/v22n6a14.pdf

13. Silva PRF, Cardoso CS, Ferreira HYS, Silva JC, Torres MTB, Silva RS, et al. Avaliação do retardo pré-hospitalar no cuidado ao infarto agudo do miocárdio no Centro Oeste de Minas Gerais, Brasil. Rev Méd Minas Gerais [serial on the internet]. 2015 [cited 2018 Nov 28];25(3):353-62. Available from: file:///D:/v25n3a08.pdf

14. Franken M. Avaliação das variáveis de desempenho no tratamento das síndromes isquêmicas miocárdicas estáveis no Brasil: análise do registro BRACE (Brazilian Registry in Acute Coronary Syndromes) [thesis on the internet]. São Paulo: Universidade de São Paulo; 2016 [cited 2018 Nov 28]. Available from: file:///D:/MarceloFranken.pdf

15. Marcolino MS, Brant LCC, Araujo JG, Nascimento $B R$, Castro LRA, Martins $P$, et al. Implantação da linha de cuidado do infarto agudo do miocárdio no Município de Belo Horizonte. Arq Bras Cardiol [serial on the internet]. 2014 [cited 2018 Nov 28];100(4):307-14. Available from: http://www. scielo.br/pdf/abc/v100n4/aop5145.pdf

16. Huguenin FM, Pinheiro RS, Varnier RM, Almeida $R$, Infantosi AFC. Caracterização dos padrões de variação dos cuidados de saúde a partir dos gastos com internações por infarto agudo do miocárdio no Sistema Único de Saúde. Rev Bras Epidemiol [serial on the internet]. 2016 [cited 2018 Nov 28];19(2):22942. Available from: http://www.scielo.br/pdf/ rbepid/v19n2/1980-5497-rbepid-19-02-00229.pdf

17. Mussi FC, Passos LCS, Menezes AA, Caramell B. Entraves no acesso à atenção médica: vivencias de pessoas com infarto agudo do miocárdio. Rev Assoc Méd Bras [serial on the internet]. 2007 [cited 2018 Nov 28];53(3):234-9. Available from: http://www. scielo.br/pdf/ramb/v53n3/a21v53n3.pdf

18. Caluza ACV, Barbosa AH, Gonçalves I, Oliveira $C A L$, Matos LN, Zeefried $C$, et al. Rede de infarto com supradesnivelamento de ST: sistematização em 205 casos diminui eventos clínicos na rede pública. Arq Bras Cardiol [serial on the internet]. 2012 [cited 2018 Nov 28];99(5):1040-8. Available from: http:// www.scielo.br/pdf/abc/v99n5/aop09512.pdf
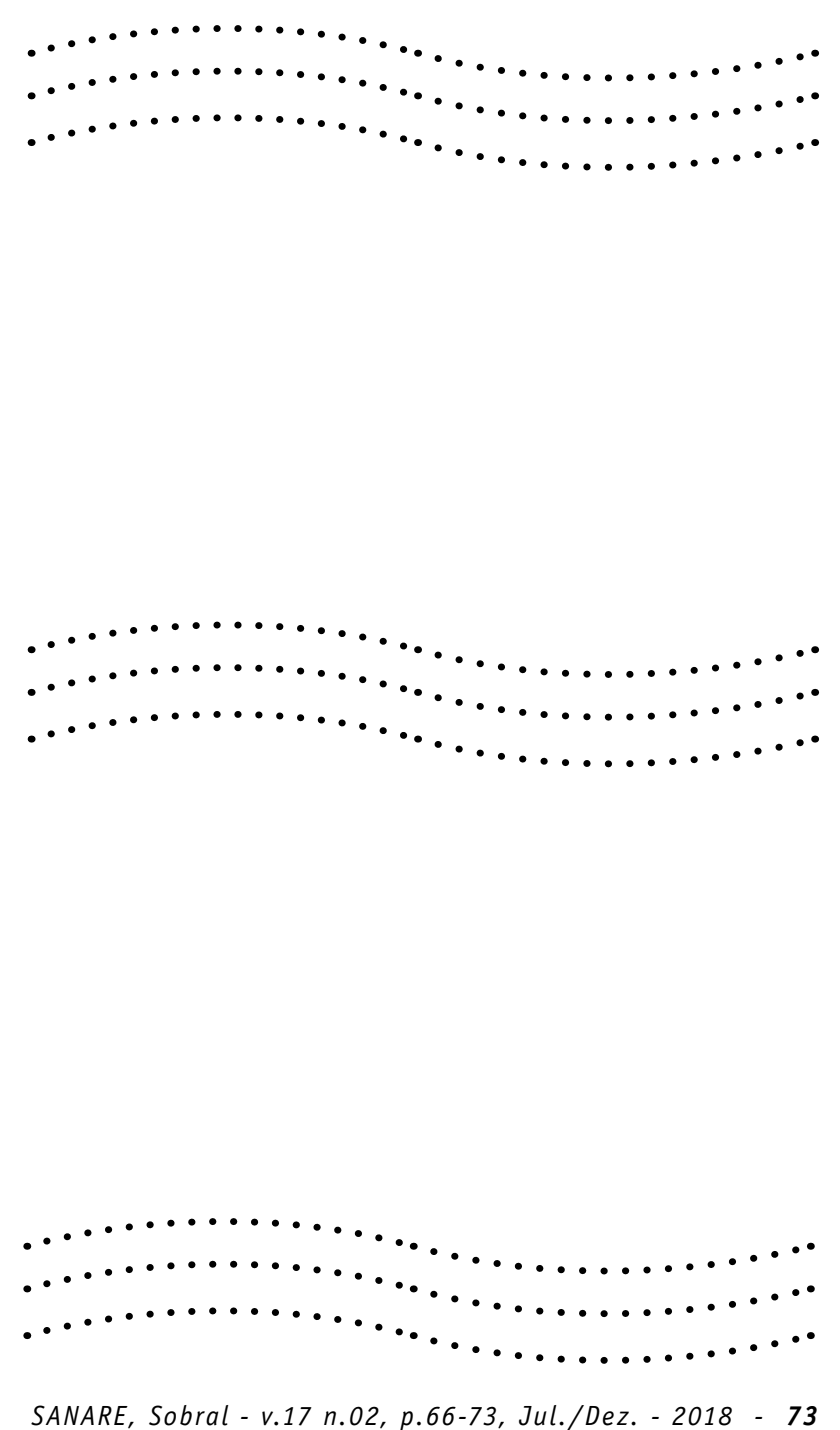